\title{
Propriedades físicas dos fios de sutura usados na odontologia
}

\section{Physical properties of suture threads used in dentistry}

\section{Humberto Lago de CASTRO}

Graduando - Faculdade de Odontologia - Universidade de Passo Fundo - Passo Fundo - RS-Brasil

\author{
Álvaro DELLA BONA \\ Professor Titular - Faculdade de Odontologia - Universidade de Passo Fundo - Passo Fundo - RS-Brasil \\ Valmor Júnior Barbosa ÁVILA \\ Professor Adjunto - Faculdade de Odontologia - Universidade de Passo Fundo - RS-Brasil
}

\begin{abstract}
Resumo
Objetivo: investigar as características físicas dos fios de sutura freqüentemente usados na odontologia. Material e Método: Fios (n=10) de seda (FS) 4-0 e nylon (FN) 4-0 (Somerville Ltda.) foram testados de acordo com a norma NBR 13904:2003. O comprimento foi determinado com escala rígida, sem tensionar o fio. O diâmetro foi avaliado utilizando um metroscópio e um relógio comparador digital. As cargas de ruptura em tração sobre nó $\left(\mathrm{L}_{\mathrm{no}}\right)$ e ao encastoamento $\left(\mathrm{L}_{\mathrm{f}}\right)$ foram testados em uma máquina de ensaio universal com velocidade de $1 \mathrm{~mm} / \mathrm{min}$. Os resultados foram analisados estatisticamente pelo teste t de Student $(\alpha=0,05)$. Resultados: Todos os fios cumpriram com as especificações quanto ao comprimento. Os FN apresentaram um valor médio de diâmetro de $0,17 \mathrm{~mm}$, contudo os FS apresentaram um valor médio de $0,21 \mathrm{~mm}$, ficando fora da faixa específica $(0,15-0,199 \mathrm{~mm})$. Valores médios de $\mathrm{L}_{\mathrm{no}}$ foram significativamente maiores para os FS $(7,54 \pm 0,50 \mathrm{~N})$ do que para os FN $(6,67 \pm 0,83 \mathrm{~N})(\mathrm{p}<0,05)$. Todos os fios resistiram ao teste de encastoamento com valores acima dos limites individual e médio mínimos preconizados, respectivamente $2,26 \mathrm{~N}$ e 4,41 N, sendo que os valores médios de FS $(8,98 \pm 2,16 \mathrm{~N})$ foram significativamente maiores do que os FN $(7,27 \pm$ $1,31 \mathrm{~N})(\mathrm{p}<0,05)$. Conclusão: Essas diferenças podem ter sido induzidas pelo maior diâmetro dos FS, pois assumindo os valores médios de diâmetro e carga obtem-se valores de resistência a tração sobre nó $\left(\sigma_{\mathrm{no}}\right)$ de 203,8 MPa para os FS e 277,9 MPa para os FN.
\end{abstract}

\section{UNITERMOS}

Propriedades físicas; fios de sutura; materiais dentários.

\section{INTRODUÇÃo}

Em cirurgia, entende-se por sutura, síntese ou síntese cirúrgica um conjunto de manobras que o cirurgião emprega para aproximar ou reunir os tecidos que foram divididos ou separados pelo ato cirúrgico ${ }^{4}$.

Para a realização da síntese são necessários fios de sutura, cujas características ideais são: segurança no nó, adequada resistência à tração, fácil manuseio, baixa reação tecidual, não possuir ação carcinogênica, não provocar ou manter infecção, manter as bordas da ferida aproximadas até pelo menos a fase proliferativa da cicatrização, ser resistente ao meio no qual atua e baixo custo ${ }^{5}$. Para que essas características sejam plenamente atendidas é necessário que as propriedades físicas dos fios de sutura sejam adequadas.

Os fios cirúrgicos têm sua numeração expressa pelo sistema USP (Farmacopéia dos Estados Unidos) como 2-0, 3-0, etc., sendo bastante variável a relação entre a numeração do fio e seu diâmetro correspondente em milímetros. Há um valor mínimo e máximo na dependência do número, como por exemplo: o número 0 varia de $0,35 \mathrm{~mm}$ até $0,40 \mathrm{~mm}$ de diâmetro do fio; o fio número $2-0$ varia entre $0,30 \mathrm{~mm}$ e $0,34 \mathrm{~mm}$ e assim por diante ${ }^{1,2}$ (Tabela 1). 
O desenvolvimento tecnológico tem possibilitado aos cirurgiões utilizarem fios de sutura com características e propriedades cada vez mais próximas das ideais. Por outro lado, o controle sobre a produção industrial dos fios de sutura, por meio de testes e a fiscalização contínua da veracidade dos resultados, é difícil de ser realizada, principalmente, devido aos altos custos operacionais ${ }^{11}$. Em virtude da carência de informações sobre tais propriedades físicas, a qualidade dos fios de sutura usados na odontologia pode estar inadequada.

Existem poucos relatos na literatura sobre as propriedades dos fios de sutura. Sardenberg et al ${ }^{11}$ (2003), avaliaram propriedades mecânicas e dimensões de fios de sutura utilizados em cirurgias ortopédicas. Fios de nylon, poliéster trançado e polipropileno de sete marcas comerciais foram submetidas a análise de diâmetro, comprimento, resistência ao encastoamento, resistência à tração do fio com nó e sem nó. Este estudo comprovou que a maioria dos fios testados encontravam-se dentro dos valores preconizados pela Associação Brasileira de Normas Técnicas (ABNT) ${ }^{1}$.

Moraes et $\mathrm{l}^{8}$ (2003), também avaliaram propriedades mecânicas (limite máximo, limite de elasticidade, rigidez, resiliência) de três fios de sutura utilizados no reparo de tendão do músculo flexor profundo do dedo de cães, porém não comparou com os valores de referência da norma NBR 13904-2003'.

Assim, esse estudo tem o objetivo de investigar as características físicas (comprimento, diâmetro, resistência à tração e encastoamento) dos fios de sutura utilizados com mais freqüência na odontologia, testando a hipótese de que as propriedades examinadas se encontram em acordo com a norma NBR 13904-2003ㄹ․

\section{MateriaIS e mÉtodos}

Foram estudados dois tipos de fio de sutura: fio de nylon 4-0 (FN) e seda 4-0 (FS) (ambos da Somerville Ltda, Jaboatão dos Guararapes, PE, Brasil). Os fios foram agrupados $(n=10)$ aleatoriamente e submetidos aos ensaios determinados pela norma NBR 139042003 e que são:

\section{Comprimento do fio}

O comprimento do fio para sutura foi determinado sobre uma superfície plana usando uma escala rígida e sem tensionamento do fio. Para os fios não absorvíveis sintéticos, o comprimento não deve ser menor que $95 \%$ do comprimento nominal ${ }^{1}$. Para os fios de sutura 4-0 não deve ser menor que $427,5 \mathrm{~mm}$.

\section{Diâmetro do fio}

Para a avaliação do diâmetro foi utilizado o metroscópio JENA (CarlZeiss, Alemanha) e um relógio comparador digital modelo ID-F150E (Mitutoyo, Japão).

As medidas foram realizadas imediatamente após a remoção do fio de sua embalagem individual mantendo-o tensionado com um peso morto de 375 g. O diâmetro foi medido em 3 pontos, aproximadamente a $1 / 4,1 / 2$ e $3 / 4$ do comprimento total, e a média das leituras foi registrada para cada fio. Os valores individuais devem estar compreendidos entre as médias dos limites para os números cirúrgicos imediatamente inferior e superior ao analisado ${ }^{1}$, o que pode ser verificado na Tabela 1.

Tabela 1 - Identificação e diâmetro de fios cirúrgicos não-absorvíveis esterilizados

\begin{tabular}{c|c|c|c}
\hline \multicolumn{2}{c|}{ Número } & \multicolumn{2}{c}{ Diâmetro $(\mathrm{mm})$} \\
\hline Métrico & Cirúrgico & Mínimo & Máximo \\
0,7 & $6-0$ & 0,070 & 0,099 \\
1,0 & $5-0$ & 0,100 & 0,149 \\
1,5 & $4-0$ & 0,150 & 0,199 \\
2 & $3-0$ & 0,200 & 0,249 \\
\hline
\end{tabular}


3. Carga de ruptura ao encastoamento $\left(L_{\mathrm{f}}\right)$ :

A finalidade deste ensaio é avaliar a fixação dos fios para sutura em agulhas atraumáticas. Esse ensaio foi realizado em uma máquina de ensaio universal (EMIC DL-2000, São José dos Pinhais, PR, Brasil) sob carga de tração até a ruptura da união fio-agulha, com velocidade de $1 \mathrm{~mm} / \mathrm{min}$. As amostras foram fixadas pela agulha em um dos prendedores da máquina, deixando a parte encastoada livre e alinhada com a direção da aplicação da força de tração. O encastoamento deve atender aos requisitos da carga mínima necessária a ruptura do encastoamento, de acordo com o diâmetro dos fios assim descritos na Tabela 2. Além disso, os fios que não romperam na região de encastoamento foram excluídos do estudo ${ }^{1}$.

Tabela 2 - Identificação dos fios cirúrgicos em agulhas atraumáticas e os valores mínimos das forças de ruptura sobre o nó e ao encastoamento

\begin{tabular}{l|c|c|c}
\hline \multirow{2}{*}{ Número cirúrgico } & $\begin{array}{l}\text { Força média mínima de ruptura } \\
\text { sob tração sobre o nó (N) }\end{array}$ & \multicolumn{2}{|c}{ Limites mínimos de força ao encastoamento (N) } \\
\cline { 2 - 4 } & Classe I & Média & Individual \\
\hline $6-0$ & 1,96 & 1,67 & 0,78 \\
$5-0$ & 3,92 & 2,26 & 1,08 \\
$4-0$ & 5,89 & 4,41 & 2,26 \\
$3-0$ & 9,41 & 6,67 & 3,33 \\
\hline
\end{tabular}

*A classe I é formada por fios de seda ou com monofilamentos de fibras sintéticas (torcidas ou trançadas), onde o possível revestimento não afeta significativamente o diâmetro. Por exemplo: seda trançada, poliéster, polipropileno, poliamida, monofilamento de poliamida ou propileno.

4. Carga de ruptura em tração sobre nó $\left(\mathrm{L}_{\mathrm{n}}\right)$ :

A força ou carga de ruptura $(\mathrm{N})$ dos fios de sutura sobre um nó simples foi mensurada usando uma máquina de ensaio universal (EMIC DL-2000, São José dos Pinhais, PR, Brasil) sob carga de tração com velocidade de $1 \mathrm{~mm} / \mathrm{min}$. Para isso, o fio foi preso pelas garras da máquina deixando o nó eqüidistante dessas. Os valores foram comparados com os de referência apresentados na Tabela 2.

No caso de rompimento em local que não no nó a amostra deveria ser excluída, caso que não ocorreu.
Todos os valores das propriedades foram analisados estatísticamente usando o teste t de Student ( $\alpha$ $=0,05)$.

\section{Resultados}

O comprimento de todos os fios examinados mostrou-se de acordo com as determinações da norma, descritas acima e apresentados na Tabela 3. Contudo, o valor médio de comprimento dos FN foi significativamente maior do que o comprimento médio dos FS $(\mathrm{p}<0,05)$.

Tabela 3 - Valores médio, mínimo e máximo de comprimento e diâmetro obtidos para os fios de sutura 4-0 examinados

\begin{tabular}{l|c|c|c|c}
\hline \multirow{2}{*}{} & \multicolumn{2}{|c|}{ Comprimento $(\mathbf{m m})$} & \multicolumn{2}{c}{ Diâmetro (mm) } \\
\cline { 2 - 5 } & FN & FS & FN & FS \\
\hline Média & $501,9 \pm 6,49$ & $457,1 \pm 2,13$ & $0,176 \pm 0,02$ & $0,217 \pm 0,14$ \\
Menor valor & 492 & 452 & 0,130 & 0,174 \\
Maior valor & 516 & 460 & 0,221 & 0,247 \\
\hline
\end{tabular}


O valor médio do diâmetro dos $\mathrm{FN}(0,17 \mathrm{~mm})$ mostrou-se dentro da norma, mas 2 amostras ficaram abaixo do valor mínimo $(0,15 \mathrm{~mm})$ e outras 2 amostras ficaram acima do máximo $(0,199 \mathrm{~mm})$. Contudo, nenhum dos diâmetros observados foi menor do que a média da faixa imediatamente anterior (fio 5-0 = $0,125 \mathrm{~mm}$ ) ou maior do que a média da faixa imediatamente posterior (fio $3-0=0,225 \mathrm{~mm}$ ) a faixa do fio analisado (4-0), estando ainda assim dentro das normas (NBR 13904-2003) (Tabela 3).

Apesar do valor médio do diâmetro dos FS $(0,21$ $\mathrm{mm}$ ) ser significativamente maior do que a média do diâmetro do FN ( $\mathrm{p}<0,05)$, a média dos FS ficou fora da faixa de medidas mínima $(0,15 \mathrm{~mm})$ e máxima $(0,199 \mathrm{~mm})$ para esse fio.
Todos os fios tiveram os valores de $\mathrm{L}_{\mathrm{f}}$ acima dos limites individual e médio mínimos preconizados $(2,26 \mathrm{~N}$ e $4,41 \mathrm{~N})$ para os fios analisados, sendo que os FS $(8,98 \mathrm{~N})$ mostraram um valor médio de $\mathrm{L}_{\mathrm{f}}$ significativamente mais elevado do que os $\mathrm{FN}(7,27$ N) $(\mathrm{p}<0,05)$, assim como o valor médio de $\mathrm{L}_{\mathrm{no}}$ foi significativamente maior para os FS $(7,54 \mathrm{~N})$ do que para os FN $(6,67 \mathrm{~N})(\mathrm{p}<0,05)$, mas ambos os fios mostraram valores acima da média mínima para essa classe de fio $(5,98 \mathrm{~N})$, de acordo com a norma NBR 13904-2003 (Tabela 4).

Durante o teste de $\mathrm{L}_{\mathrm{f}}$ foram desprezados $2 \mathrm{FN}$ e 10 FS pois não romperam na região de encastoamento, ou seja, na união agulha-fio. Contudo, o valor de $\mathrm{L}_{\mathrm{f}}$ para esses fios foi acima de $10 \mathrm{~N}$.

Tabela 4 - Valores médio, desvio padrão (DP), mediana, mínimo e máximo de $L_{n o}$ e $L_{f}$ para os fios de sutura 4-0 examinados

\begin{tabular}{|c|c|c|c|c|}
\hline & \multicolumn{2}{|c|}{ Força de ruptura ao encastoamento $-L_{f}(N)$} & \multicolumn{2}{|c|}{ Força de tração sobre nó $-L_{n o}(N)$} \\
\hline & FN & FS & FN & FS \\
\hline Média \pm DP & $7,26 \pm 1,31$ & $8,98 \pm 2,16$ & $6,67 \pm 0,84$ & $7,54 \pm 0,50$ \\
\hline Mediana & 7,96 & 9,95 & 6,67 & 7,50 \\
\hline Valor Mínimo & 4,98 & 4,89 & 5,57 & 6,71 \\
\hline Valor Máximo & 8,62 & 11,57 & 8,22 & 8,38 \\
\hline
\end{tabular}

\section{Discussão}

As propriedades dos fios de sutura são divididas em características físicas, de manuseio e de reação tecidual ${ }^{5}$. O presente estudo é limitado às características físicas, particularmente às dimensões (comprimento e diâmetro), resistência ao encastoamento e resistência à tração sobre nó, as quais são descritas na norma NBR 13904-2003.

A comparação das características de dimensões observadas nesse estudo apresenta importância relativa no uso prático de fios de sutura, sendo mais relevante para o controle de qualidade da fabricação e comercialização ${ }^{11}$.

As propriedades mecânicas, especificamente a resistência ao encastoamento e a resistência à tração sobre nó são fundamentais na prática odontológica. A resistência ao encastoamento, isto é, a força necessária para romper a união entre o fio e a agulha, é importante na realização de pontos cirúrgicos, sendo fácil compreender que uma agulha que se solte do fio durante a sua passagem por um tecido, pode comprometer a técnica bem como inutilizar o restante do fio.

A necessidade de testar a resistência à ruptura do fio com nó está fundamentada no fato de que quando o cirurgião aperta o nó da sutura espera-se que não haja ruptura do fio muito menos soltura desse ponto até a fase proliferativa da cicatrização tecidual ${ }^{6,7,9,13}$.

Castro et $a l^{3}$ (1986) e Silveira \& Heitz ${ }^{12}$ (1998) descrevem os fios de sutura como causadores de irritação aos tecidos e que determinam uma resposta inflamatória de baixa intensidade e curta duração.

A reação cicatricial provocada pelos fios é proporcional à sua espessura, ou seja, uma sutura feita com um fio de diâmetro calibroso apresenta uma resposta mais intensa no tecido do que aquela realizada com o mesmo tipo de fio, porém com menor espessura ${ }^{10}$. 
Existem estudos comparando materiais de diferentes composições, no sentido de orientar os cirurgiões na escolha do fio ideal ${ }^{6,9,13,14}$. No presente estudo as comparações foram realizadas em relação aos padrões recomendados pela norma NBR 13904-2003 da ABNT, analisando os fios de sutura comumente utilizados na odontologia em relação ao padrão nacional recomendado. Essa norma não recomenda especificamente a velocidade de aplicação da carga para os ensaios mecânicos de resistência. Assim foi utilizado a velocidade de $1 \mathrm{~mm} / \mathrm{min}$, com uma célula de carga compatível (200 N) e $35 \mathrm{~mm}$ de distância entre uma garra e outra da máquina de ensaio universal para o teste de encastoamento e $50 \mathrm{~mm}$ de distância para o teste de resistência à tração sobre nó. Assim, os resultados do presente estudo foram comparados aos padrões da norma NBR 13904 da ABNT, o que indicou que todas as propriedades dos fios testados estão de acordo com a norma, exceto pelo diâmetro médio dos FS $(0,217 \mathrm{~mm})$ ficou acima do valor médio máximo recomendado para esse tipo de fio $(0,199 \mathrm{~mm})$, o que provavelmente induziu a valores de $\mathrm{L}_{\mathrm{f}}$ e $\mathrm{L}_{\mathrm{no}}$ maiores para esse fio (FS). Isso pode ser verificado em uma simulação do cálculo de resistência para essas duas propriedades, ou seja, a resistência a tração sobre nó $\left(\sigma_{\text {no }}\right)$ e a resistência ao encastoamento $\left(\sigma_{\mathrm{f}}\right)$, que é a razão entre as cargas médias $(\mathrm{L} \mathrm{em} \mathrm{N})$ e a área transversal dos fios $\left(\mathrm{A}=\mathrm{r}^{2} \pi \mathrm{em} \mathrm{m^{2 }}\right)$. Assim, assumindo os valores médios de diâmetro e carga obtem-se valores de $\sigma_{\text {no }}$ de 203,8 MPa para os FS e 277,9 MPa para os FN, e valores de $\sigma_{\mathrm{f}}$ de 242,7 MPa para os FS e 302,5 MPa para os FN. Portanto, a norma 13904 menciona os termos resistência ao encastoamento e a tração sobre o nó, mas na realidade descreve procedimentos técnicos para obtenção da força ou carga máxima de ruptura (em $\mathrm{N})$, onde o correto para propriedades de resistência $(\sigma=\mathrm{L} / \mathrm{A})$ seria resultados em unidades de MPa. Esse raciocínio justifica o tratamento dado a essas propriedades nesse estudo.

Sardenberg et $a l^{11}$ realizaram testes semelhantes, porém com outras marcas comerciais e também verificou que algumas estavam com a média do diâmetro da amostra fora da faixa preconizada para os fios 4-0. As duas marcas estudadas (Point Suture e Ethicon) apresentaram diâmetro maior para os $\mathrm{FN}$ que o recomendado, o que diverge deste estudo, onde os FN (Somerville) estavam dentro do diâmetro recomendado.

A qualidade dos fios de sutura utilizados na odontologia pode estar comprometida, uma vez que há uma carência de estudos científicos bem delineados e a fiscalização da produção desse produto é difícil de ser implementada. O que foi confirmado parcialmente neste estudo, com o diâmetro os FS acima da média e as devidas implicações discutidas acima. Assim, é necessário que outros estudos verifiquem outras marcas comerciais e suas propriedades, comparando-as com as normas da ABNT.

\section{Conclusão}

Baseado nos resultados deste estudo, conclui-se que os fios de seda e nylon 4-0 da marca Somerville Ltda estão dentro dos padrões descritos na norma NBR 13904:2003. Porém o fio de seda apresentou uma média de diâmetro acima do recomendável, o que possivelmente influenciou nos resultados mais elevados de tração sobre nó e ao encastoamento.

\begin{abstract}
Objective: to investigate the physical properties of suture threads often used in dentistry. 4-0 silk threads (FS) and 4-0 nylon threads (FN) were tested ( $n=10)$ according to the NBR 13904:2003 standard. Material and Methods: The length was determined with a fixed scale, without stretching. For the evaluation of the diameter a measuring microscope and a digital caliper were used. The maximum tensile load on knot $\left(L_{n}\right)$ and the maximum needle-to-thread joint load $\left(L_{f}\right)$ were tested in a universal testing machine with a crosshead speed of $1 \mathrm{~mm} / \mathrm{min}$. The results were analyzed using the Student $t$ test $(\alpha=0.05)$. Results: All threads were in agreement with the length specifications. The mean diameter value was $0.17 \mathrm{~mm}$ for the FN and $0,21 \mathrm{~mm}$ for the $F S$, which is not within the specifications $(0.150-0.199 \mathrm{~mm})$. The mean $L_{n o}$ value of FS $(7.54 \pm 0.50 \mathrm{~N})$ was significantly greater than the mean $L_{n o}$ value of $F N(6.67 \pm 0.83 \mathrm{~N})(p<0.05)$. All specimens showed the $L_{f}$ values above the minimum individual and mean values required by the standard, which are $2.26 \mathrm{~N}$ and $4.41 \mathrm{~N}$ respectively, where the mean $L_{f}$ value of $F S(8.98 \pm 2.16 N)$ was significantly greater than the mean $L_{f}$ value of $F N(7.27 \pm 1.31 N)(p<0.05)$. Conclusion: These differences could have been induced by the greater diameter value of the FS, since assuming the mean diameter and maximum load values resulted in mean tensile strength on knot $\left(\sigma_{n o}\right)$ of 203,8 MPa for FS and 277,9 MPa for FN.
\end{abstract}

\title{
UNITERMS
}

Physical properties; suture threads; dental materials. 


\section{REFERÊNCIAS}

1. Associação Brasileira de Normas Técnicas. NBR 13904; Fios para sutura cirúrgica. Rio de Janeiro; 2003. 15p.

2. Bellen BV, Magalhães HP. Técnica cirúrgica e cirurgia experimental. São Paulo: Ed. Sarvier; 1989.

3. Castro HL, Okamoto T, Castro AL. Fios de sutura sintéticos. Estudo histológico em ratos. Ars cvrandi odontol. 1978; 5:32-42.

4. Graziani M. Técnica Cirúrgica. In. Cirurgia Buco-maxilo-facial. Rio de Janeiro: Guanabara Koogan; 1986.

5. Hering FLO, Gabor S. Bases técnicas e teóricas dos fios de sutura. São Paulo: Ed. Roca; 1993.

6. Hermann JB. Tensile strength and knot security of surgical suture materials. Am Surg. 1971; 37: 209-17.

7. Holmlund DEW. Knot properties of surgical suture materials. Acta Chir Scand. 1974; 140: 355-62.

8. Moraes JRE, Favaro AF, Shimano AC, Ferraro GC, Moraes FR. Propriedades mecânicas de três fios de sutura no reparo do tendão do músculo flexor profundo do dedo de cães. Braz J Vet Res, Anim Sci, 2003; 40: 443-451.

9. Muller SS, Sardenberg T, Danieli MV, Pizol F. Avaliação biomecânica de sutura tendinosa com 3 tipos de fios cirúrgicos: estudo experimental em cães. In: III Congresso Gaúcho de Ortopedia e Traumatologia. Porto Alegre, 2002.
10. Peterson LJ, Ellis E, Hupp JR, Tucker MR. Cirurgia oral e maxilofacial. Rio de Janeiro: Elservier Editora; 2005.

11. Sardenberg T, Muller SS, Silvares PRA, Mendonça AB, Moraes RRL. Avaliação das propriedades mecânicas e dimensões de fios de sutura utilizados em cirurgias ortopédicas. Acta Ortop Bras. 2003; 11(2):88-94.

12. Silveira JOL, Heitz C. Instrumental, materiais, equipamentos e ambiente cirúrgico. In: Silveira JOL, Beltrão GC. Exodontia. Porto Alegre: Missau; 1998.

13. Trail IA, Powell ES, Noble J. An evaluation of suture materials used in tendon surgery. J Hand Surg Br. 1989; 14: 422-7.

14. Wright PE. Lesões dos tendões flexores e extensores. In: Crenshaw AH. Cirurgia Ortopédica de Campbell. São Paulo: Manole; 1997.

Recebido em: 18/06/07

Aprovado em: 30/07/07

Endereço para correspondência: Álvaro Della Bona

Faculdade de Odontologia da Universidade de Passo Fundo BR 285 - Bairro São José - Campus I - Cx Postal 611

Telefone (54) 33168402 - CEP: 99001-970 - Passo Fundo - RS 